\title{
European Society of Child and Adolescent Psychiatry: position statement on mental health of child and adolescent refugees
}

\author{
Dimitris C. Anagnostopoulos ${ }^{1} \cdot$ Johannes Heberbrand $^{2} \cdot$ Stephan Eliez $^{3} \cdot$ \\ Maeve B. Doyle ${ }^{4} \cdot$ Henrikje Klasen $^{5} \cdot$ Sofie Crommen $^{6} \cdot$ Fusun Cetin Cuhadaroğlu $^{7}$. \\ Milica Pejovic-Milovancevic ${ }^{8} \cdot$ Oscar Herreros $^{9} \cdot$ Ruud Minderaa $^{10}$. \\ Andreas Karwautz $^{11}$ • Carl Goran Svedin ${ }^{12} \cdot$ Jean Philippe Raynaud $^{13}$
}

Published online: 29 June 2016

(C) Springer-Verlag Berlin Heidelberg 2016

\section{Introduction}

The reaction of the European Society of Child and Adolescent Psychiatry (ESCAP) to the increasing numbers of refugees entering Europe was to initiate the project titled ESCAP for mental health of child and adolescent refugees, to be carried out by members of the ESCAP board and a group of volunteers. The aims and mission of this initiative were published as an editorial in the January 2016 issue of
ECAP [1]. Since then, a number of activities have been put in place to organize the knowledge to support mental health care for refugee children and make it available everywhere in Europe.

The ESCAP online forum (http://www.escap.eu/care/ position-statement-announcement/escaps-approach/), introduced 6 months ago to openly discuss challenging issues and allow professionals to share their experience, has served as a valuable platform to gather, enrich and

Jean Philippe Raynaud

raynaud.jp@chu-toulouse.fr

danagnost@med.uoa.gr

Johannes Heberbrand

johannes.hebebrand@uni-due.de

Stephan Eliez

stephan.eliez@etat.ge.ch

Maeve B. Doyle

MaeveB.Doyle@hse.ie

Henrikje Klasen

henrikje.klasen@gmail.com

Sofie Crommen

sofiecrommen@hotmail.com

Fusun Cetin Cuhadaroğlu

fusun.cetin@gmail.com

Milica Pejovic-Milovancevic

milica.pejovic@imh.org.rs

Oscar Herreros

oherreros@gmail.com

Ruud Minderaa

r.minderaa@gmail.com

Andreas Karwautz

andreas.karwautz@meduniwien.ac.at

Carl Goran Svedin

carl.goran.svedin@liu.se
1 Department of Child and Adolescent Psychiatry, School of Medicine, National and Kapodistrian University of Athens, Athens, Greece

2 Department of Child and Adolescent Psychiatry, University of Duisburg-Essen, Essen, Germany

3 Department of Psychiatry, School of Medicine, University of Geneva, Geneva, Switzerland

4 Department of Child and Adolescent Psychiatry Cavan/ Monaghan Mental Health Service, St Davnet's Hospital, Roosky, Co. Monaghan, Ireland

5 Medical Centre, Leiden University, Leiden, Netherlands

6 Flemish Association for Child and Adolescent Psychiatry, Antwerp, Belgium

7 Department of Child and Adolescent Psychiatry, School of Medicine, Hacettepe University, Ankara, Turkey

8 Clinic for Child and Adolescent Psychiatry, School of Medicine, Belgrade University, Belgrade, Serbia

9 Hospital Universitario Virgen de las Nieves, Granada, Spain

10 European Society of Child and Adolescent Psychiatry, Antwerp, Belgium 
distribute knowledge for anybody involved in the mental health care for refugee children. Background information, relevant articles published in ECAP and a review on unaccompanied refugee minors are readily available online. Also, several practical documents provide clinical guidance for child and adolescent mental health in refugees and migrants. Various instruments, such as evidence-based guidelines, video modules, manuals, guides for parents and their supporters, make the guidance available to different audiences (mental health workers and non-professional volunteers, psychologists, social workers, and child and adolescent psychiatric services). ESCAP members have also published background articles and lectured at scientific meetings with a focus on mental health issues of child and adolescent refugees. Thankfully, the team of volunteers has grown, and the project will go forward. A special ECAP issue including both original articles and reviews scheduled for 2017 further underscores our efforts to provide mental health professionals with an in-depth insight into this ongoing challenge.

ESCAP is well aware of its responsibility and the challenging work lying ahead. As a result of our experience so far, we have worked together to devise an ESCAP position statement calling for action.

\section{Preamble}

Flight and migration are not new phenomena, and many countries in Europe and the Middle East have been experiencing the recent refugee/migration wave. The number of refugees coming to Europe has currently reached staggering proportions in some countries, and it is, to some degree, unpredictable as to what extent, and in which countries, this influx will increase or decrease. The brunt of the refugee influx has been borne by those countries in eastern, southern, middle, and northern Europe that the refugees travel through and finally settle in. A successful mediumand long-term integration is fraught with uncertainty due to the possibility of refugees returning (or having to return) to their home countries and the potential development of tensions between subgroups of both the refugees and the local and/or national populations.

In the EU-28 (total population 508 million inhabitants), first-time asylum applicants in 2015 were 1,255,688, $29 \%$

11 Department of Child and Adolescent Psychiatry, Medical University of Vienna, Vienna, Austria

12 Department of Child and Adolescent Psychiatry, Linköping University, Linköping, Sweden

13 Centre Hospitalier Universitaire de Toulouse, Toulouse, France of whom were minors $(19 \%$ <age $14 ; 10 \%$ between 14 and 17 years). Indeed, the numbers of asylum applicants do not capture the scope of the problem, since a large proportion of those fleeing or migrating from conflict zones opt for a residence permit or refuse to apply for either option.

The high prevalence of physical disorders and nutritional deficiencies among young refugees warrant attention, and provision of somatic health care represents a major concern particularly during the flight and upon arrival at the country of settlement.

Child and adolescent refugees are exposed to many risks pre-flight, during their flight, and upon arrival, which make them also vulnerable for the development of psychiatric disorders, such as post-traumatic stress disorder (PTSD), anxiety disorders, mood disorders or externalizing disorders.

The pre-flight experiences of young refugees depend on their country of origin; exposures to poverty, war, or warlike conditions are common. Also, the acquired education, social status, familial, religious, and sociocultural values also shape coping and help-seeking behavior.

The flight in itself can be traumatic or compound trauma via, for instance, separation experiences, sexual abuse, and trafficking including forced labor and sexual exploitation. In this context, it should be pointed out that many unaccompanied underage refugees attempt to travel in groups, which include adults known to them; in many cases, the parents of unaccompanied children and adolescents are aware of their travels. To allow for better protection of such young refugees, it would seem helpful not to totally separate underage refugees from such groups.

The arrival in the hosting country entails risks due to unsafe or otherwise problematic living conditions, nonaccess to schooling, years of insecurity with uncertain legal and residential status, multiple moves, parental illness and unemployment, social exclusion, and in the mediumand long-term maladaptation with respect to the cultural norms of the hosting country. Hostility toward foreigners and refugees, in particular, represents a threat that requires both surveillance of refugee camps and political education within the hosting country. Within this context, the initial provision of a safe environment to traumatized young refugees should not be taken for granted. Moreover, even once migrants have settled and formed families, their children, the second-generation migrants, have an increased risk for mental health problems.

European attitude toward young refugees and their families will greatly determine the burden of trauma, not only on their adult future but also on our community. An empathic and mentalizing attitude, secure sheltering, addressing health and educational needs will create a sense of stability and confidence. This is the very first step to favor, for these future adults and their families, either a 
productive integration in the European heritage of strength and diversity, or the potential to rebuild and stabilize their native countries for those who will return.

\section{ESCAP call for action}

- The European Society for Child and Adolescent Psychiatry (ESCAP) calls upon all governments and political groups with influence in the regions of conflict and war to draft solutions to resolve these conflicts and bring an end to the present refugee crisis. The physical and mental health of children and of future generations in these countries is further compromised by prolongation of these conflicts.

- ESCAP calls for all basic health care to be provided to migrants, with a joint focus on children's physical and mental health. The interventions should follow the different options, needs and peculiarities that every country is faced with. The different stages of the refugee flight and resettlement process have to be taken under consideration.

- Organizations (including governmental) should be encouraged to release professionals to work with these populations; training of such professionals with respect to the needs of migrants is important.

- Activities of all professionals and organizations working with children in these circumstances must apply the principles of best interests identified in the UN convention on the rights of the child (CRC; article 3). Further, the rights laid down in the CRC should be upheld regardless of the child's immigration status. This applies to all children up to the age of 18 years.

- All those working with children, adolescents and their families, should safeguard their rights to be heard and to participate in decisions that concern them.

- Hosting countries should particularly try to make the steps leading to a legalization of the residential status and the granting of asylum as transparent as possible. This requires provision of information on the time required for decision processes and a basic understanding of how decisions are made. An acceleration of such processes will help the youth to adjust in a better way.

- Children should not be separated from their families as long as this is consistent with their best interests. Existing relationships, including those between youths who have strong bonds through shared experiences, should be respected. If the quality of the relationship is deemed trustworthy, the same applies to bonds between unaccompanied youths and adults.

- Financial resources of every country hosting a larger number of refugees are strained. Thus, optimal use of funding is crucial. Investments are initially primarily required for provision of a safe environment, appropriate schooling, and the youth welfare system. The promotion of a healthy adaptation of these young people and their families and lowering their risks are crucial. More European support is required particularly for less welloff countries with a high number of refugees.

- Children and youth reaching destination countries should be supported to integrate into and be provided with mainstream services as well as the regular education system in a non-discriminatory and culturally sensitive way. At the same time, they should be assessed for and provided with any necessary additional support. A successful educational, cultural, and, if applicable, religious integration is the key cornerstone for future mental health and the prevention of behavioral disorders.

- Adolescent refugees who turn 18 and who have started an education/apprenticeship should be allowed to temporarily stay in the respective country to enable them to return to their home country as well-educated individuals.

- Young refugees with developing or preexisting serious mental disorders need to be identified to ensure access to mental health care. ESCAP through its member societies can contribute to this goal.

- To meet the current challenge, we need to adopt a public health approach making more use of screening, stepped care, task sharing, and task shifting in our current structures, health care financing, and ways of working. Enhancing cultural competence of professionals and monitoring refugees' access and utilization of services are also needed.

- We should give a special emphasis toward understanding refugees' experiences and challenges within the new environment and toward fostering resilience among individuals and communities. We need to build up and make use of model communities, which have programs in place to promote resilience and integration/assimilation.

- It is crucial to meet the needs of all youths, native and immigrants, without any discrimination between them.

- ESCAP calls on all stakeholders to gather and distribute state-of-the-art knowledge which mainly focuses on the acute needs of refugees, the risk and protective factors for their mental health, and the specific interventions that are needed, taking into consideration the different needs and available resources of the various EU countries.

European attitude toward young refugees and their families will greatly determine the burden of trauma and attachment disorders, not only on their adult future but also on our community. An empathic and mentalizing attitude, secure sheltering, addressing health and educational needs will create a sense of stability and confidence. This 
is the very first step to favor, for these future adults and their families, either a productive integration in the European heritage of strength and diversity, or the potential to rebuild and stabilize their native countries for those who will return.

\section{Reference}

1. Hebebrand J, Anagnostopoulos D, Eliez S, Linse H, PejovicMilovancevic M, Klasen H (2016) A first assessment of the needs of young refugees arriving in Europe: what mental health professionals need to know. Eur Child Adolesc Psychiatry 25:1-6 